\title{
lodine nutrition of pregnant and lactating women in Hong Kong, where intake is of borderline sufficiency
}

\author{
Annie WC Kung* \\ Department of Medicine, The University of Hong Kong, Queen Mary Hospital, Hong Kong SAR, China
}

\begin{abstract}
Objective: To describe the iodine nutrition of pregnant and lactating women in Hong Kong, where intake is of borderline sufficiency.

Design: Review of cross-sectional and prospective studies.

Setting: China, Hong Kong Special Administrative Region (SAR).

Subjects: Pregnant and lactating women.

Results: Studies of pregnant women in Hong Kong SAR have revealed an increase in the urinary iodine (UI) concentration as pregnancy advances. A significant percentage of women had a sub-normal serum thyroid hormone concentration at full term. Although iodine is concentrated by the mammary gland, 19\% of all mothers had low iodine concentrations in their breast milk. The moderate correlation between the concentrations of iodine in breast milk and urine suggests that an adequate maternal urinary iodine concentration cannot reliably indicate that an infant is getting enough iodine in breast milk. Therefore, some breast-fed infants may still be at risk of low iodine intake, and additional iodine supplements, other than salt iodisation, would be warranted in this population.

Conclusions: The currently recommended intake of iodine through universal salt iodisation may not be adequate for pregnant and lactating women, and supplementation during pregnancy and lactation should be further considered in light of the latest recommendations.
\end{abstract}

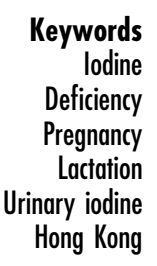

An adequate iodine intake during pregnancy and lactation is important because both the foetus and the neonate are particularly vulnerable to the harmful effects of deficiency. The clinical consequences of severe iodine deficiency (ID) are easily recognised, but the effects of mild to moderate deficiency are often overlooked: thyroid enlargement in both the mother and the neonate, and hypothyroidism, hyperthyrotropinaemia and impaired neuropsychological development of the neonate ${ }^{1}$.

In localities in which the iodine intake of the general population is adequate because of complete or partial salt iodisation programmes, the problem of ID in pregnant and lactating women, and hence in neonates, is minimal. Even in countries with a recently introduced but well-monitored salt iodisation programme, such as China, the goitre rate is low at about $5 \%$, and the median urinary iodine (UI) concentrations of women of reproductive age and those who are pregnant and lactating indicate an adequate iodine nutrition ${ }^{2}$. This suggests that salt iodisation can prevent ID not only in schoolchildren, but also in pregnant mothers and their neonates. A randomised controlled study in China confirmed that infants born to mothers supplemented with iodised salt had significantly better gross motor function, language skills and development quotient, and that the percentage of infants born with a sub-normal development quotient was significantly lower than in controls ${ }^{3}$. A study in an area with an effective salt iodisation programme among the general population found that infants born with a concentration of thyroid-stimulating hormone (TSH) in cord blood of less than $5 \mathrm{mIU}^{-1}$ had significantly better mental and psychomotor development, better information processing skills and better cognitive development than infants with a cord blood TSH concentration above $10 \mathrm{mIU}$ per $\mathrm{l}^{4}$.

Apart from salt iodisation, other types of iodisation programmes can also be effective in preventing iodine deficiency disorders (IDD) in infants. For example, the iodisation of irrigation water in remote villages in Xinjang Province, China effectively and greatly reduced the neonatal and infant mortality rate ${ }^{5,6}$. Similarly, iodised oil given orally to women prior to and during pregnancy decreased the total goitre rate and the percentage of infants born with abnormal intelligence when compared with a control group ${ }^{7}$.

It is generally agreed that pregnant and lactating women require a greater iodine intake than non-pregnant women, and the ICCIDD, the UNICEF and the WHO recommend a daily intake of $200 \mu \mathrm{g}^{8}$. As there is no reference indicator to define ID in pregnant and lactating women, it is difficult to monitor iodine status in both the pregnant mothers and their offspring. It has been reported that an intake of more than $1000 \mu \mathrm{g}$ iodine per day by pregnant women can result in 
hypothyroidism and hyperthyrotropinaemia of neonates 9 . Babies born to mothers with a UI concentration of $<175$ or $>500 \mu \mathrm{gl}^{-1}$ were more likely to have a cord blood TSH concentration above $10 \mathrm{mIUl}^{-1}$ and a low birth weight ${ }^{10}$. However, it can be difficult to interpret the cord blood TSH concentration as it is affected by stress during delivery.

In places where there is no salt iodisation programme, it is uncertain whether the median UI concentration of schoolchildren and their total goitre rate can adequately reflect the iodine nutrition of other groups who may be at risk of deficiency, such as pregnant and lactating women. The experience in Hong Kong, where iodine intake is of marginal or borderline sufficiency, suggests that monitoring schoolchildren alone is not adequate to prevent ID in pregnant and lactating women and their offspring. In Hong Kong, a special administrative region in southern China, the median UI in schoolchildren was above $100 \mu \mathrm{gl}^{-1}$ and less than $20 \%$ of urine samples had an iodine concentration below $50 \mu \mathrm{g}$ per $1^{11,12}$. The total goitre rate among the schoolchildren was $3 \%{ }^{13}$. These data suggest that schoolchildren in Hong Kong have an adequate iodine intake. However, neonatal screening has shown that the median cord blood TSH is $5.6 \mathrm{mIUl}^{-1}$ and $22 \%$ of the cord blood samples had a TSH concentration of $>10$ mIU per $1^{14}$. The incidence of neonatal hypothyroidism was 1 in 3300 and $23 \%$ of cases of congenital hypothyroidism were transient in nature ${ }^{14}$.

Cross-sectional and prospective studies of pregnant women in various trimesters of pregnancy in Hong Kong have revealed an increase in the UI concentration as pregnancy advances ${ }^{15}$. With a median UI of approximately $100-120 \mu \mathrm{gl}^{-1}$ during the various stages of pregnancy, a significant percentage of women had a sub-normal serum thyroid hormone concentration at full term: 63\% had a low free tri-iodothyronine concentration, 53\% had a low thyroxine concentration and 5\% had a low free thyroxine index. The thyroid gland was found to increase in volume by $30 \%$ during pregnancy and $25 \%$ of women had a gland size greater than 2 standard deviations of the mean size for non-pregnant southern Chinese women ${ }^{15}$. Pregnant women starting their second trimester with excessive thyroid stimulation, as shown by a high serum thyroglobulin concentration and a low free thyroxine concentration, had a low median UI and a large thyroid volume throughout their pregnancy. One half of the neonates born to these women had a sub-normal free thyroxine concentration at birth. It thus appears that a median UI of approximately $100-120 \mathrm{gl}^{-1}$ during pregnancy is associated with maternal hypothyroxinaemia and thyroid enlargement, as well as hypothyroidism of neonates. Furthermore, women with excessive UI loss during pregnancy are more likely than others to develop new thyroid nodules during the second and third trimester of pregnancy ${ }^{16}$.

In places without a salt iodisation programme, iodine supplementation of pregnant and lactating women and monitoring the UI concentration are necessary to prevent neonatal hypothyroidism and maternal goitrogenesis. Iodine supplements should ideally be given to women of reproductive age before conception to replenish their thyroid iodine store and thus ensure optimal early foetal brain development.

\section{References}

1 Stanbury JB, Delange F, Dunn JT, Pandav CS. Iodine in Pregnancy. New Delhi: Oxford University Press, 1998.

2 Specialist Guide Group of the Fourth National Survey on IDD. China National Iodine Deficiency Disorders Surveillance Report 2002. Beijing: Center for Endemic Disease Control, Chinese Center for Disease Control and Prevention, 2003.

$3 \mathrm{Hu} \mathrm{L}$, Su Z, Wang F. Study on relationship between iodinenutritional status and infants' intelligence levels. Chinese Journal of Epidemiology 1998; 17: 213-5.

4 Choudhury N, Gorman KS. Subclinical prenatal iodine deficiency negatively affects infant development in northern China. Journal of Nutrition 2003; 133: 3162-5.

5 DeLong GR, Leslie PW, Wang S, Jiang S, Zhang M, Rakeman MA, Jiang J, Ma T, Cao X. Effect on infant mortality of iodination of irrigation water in a severely iodine-deficient area of China. Lancet 1997; 350: 771-3.

6 Ren Q, Delong GR, Cao X, Wang S, Jiang X, Jiang J, Ma E, O'Donnell K. Effect of environmental supplementation of iodine on infant mortality and growth in children in Xinjiang, China. Chinese Journal of Epidemiology 2002; 23: 198-202.

7 Zheng H, Liu Y, Wang C, et al. Effect of oral iodized oil capsule in pregnant women on intelligent and physical development of filial generation. Chinese Journal of Epidemiology 1998; 17: 299-302 (Original in Chinese, English abstract seen).

8 International Council for Control of Iodine Deficiency Disorders, United Nations Children's Fund, World Health Organization. Assessment of Iodine Deficiency Disorder and Monitoring Their Elimination. A Guide for Programme Managers, 2nd ed. Geneva: World Health Organization, 2001.

9 Nishiyama S, Mikeda T, Okada T, Nakamura K, Kotan T, Hishinuma A. Transient hypothyroidism or persistent hyperthyrotropinemia in neonates born to mothers with excessive iodine intake. Thyroid 2004; 14: 1077-82.

10 Zhao J, Xie H, Mao X, Zhang X. Study on correlation of maternal iodine-nutrition and the thyroid function and the physical development of their infants. Journal of Practical Obstetrics and Gynaecology 2000; 16: 248-9.

11 Low LC, Lin HJ, Cheung PT, Lee FT, Chu SY, Kwok TL, Bacon-Shone J, Yeung CY. Screening for congenital hypothyroidism in Hong Kong. Australian Paediatric Journal 1986; 22: 53-6.

12 Wong GW, Lam CW, Kwok MY, Mak TW, Ahuja AT, Chow CC, Tang W. Children goitre and urinary iodine excretion in Hong Kong. European Journal of Pediatrics 1998; 157: 8-12.

13 Kung AWC, Chan LWL, Low LCK, Robinson JD. Existence of iodine deficiency in Hong Kong-a coastal city in southern China. European Journal of Clinical Nutrition 1996; 50: 569-72.

14 Lo KK, Lam ST. Neonatal screening programme for congenital hypothyroidism in Hong Kong. In: Lam ST, Pang CC, eds. Neonatal and Perinatal Screening: the Asian Pacific Perspectives. Hong Kong: The Chinese University Press, 1996; 145-8.

15 Kung AWC, Lao TTH, Chau MT, Tam S, Low LCK. Goitrogenesis during pregnancy and neonatal hypothyroxinaemia in a borderline iodine sufficient area. Clinical Endocrinology 2000; 53: 725-31.

16 Kung AWC, Chau MT, Lao TTH, Tan S, Low LCK. The effect of pregnancy on thyroid nodule formation. Journal of Clinical Endocrinology and Metabolism 2002; 87: 1010-4. 